\title{
Nonlinear spinor in a Kerr-Schild background
}

A. C. Cadavid, and R. J. Finkelstein

Citation: Journal of Mathematical Physics 42, 4419 (2001); doi: 10.1063/1.1388031

View online: http://dx.doi.org/10.1063/1.1388031

View Table of Contents: http://aip.scitation.org/toc/jmp/42/9

Published by the American Institute of Physics

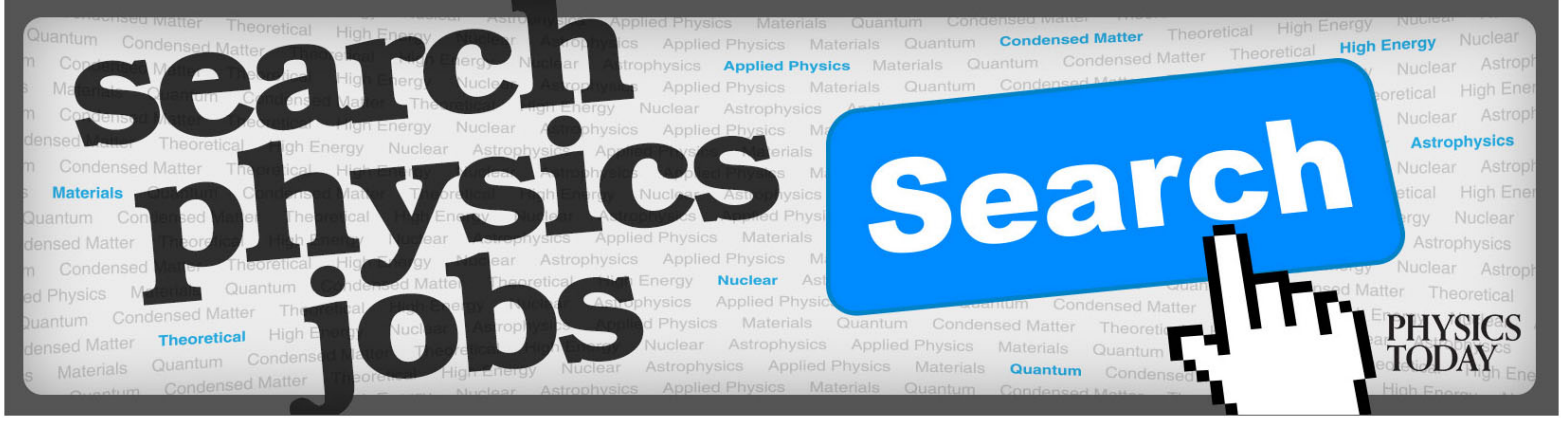




\title{
Nonlinear spinor in a Kerr-Schild background
}

A. C. Cadavid ${ }^{\text {a) }}$

Department of Physics, California State University, Northridge, California 91330

R. J. Finkelstein

Department of Physics and Astronomy, University of California, Los Angeles, California 90095-1547

(Received 31 January 2001; accepted for publication 22 May 2001)

\begin{abstract}
We study the nonlinear spinor field in a Kerr-Schild background by first looking for solitonic solutions in the absence of rotation, given that in the special relativistic limit there are solitonic solutions in this approximation. Since for the scalar field problem at least two independent radial functions are needed for the solitonic solutions to exist, we introduce a dilation field by deforming the Kerr-Schild metric by a Weyl factor. We find that for the parameter space studied there are no solitonic solutions in the spherically symmetric approximation. While in the present work we have studied the spinor field, we also had in mind a corresponding study of a scalar field in the same background where it may be of interest for describing a rotating stellar soliton. The proposed strategy in both cases begins by looking for solitonic solutions in the absence of rotation and then continues by perturbatively correcting for the rotation required by the angular dependence of the Kerr background. The strategy appears to be unsuccessful. (C) 2001 American Institute of
\end{abstract} Physics. [DOI: 10.1063/1.1388031]

\section{INTRODUCTION}

Elementary particle solitons have been extensively investigated at the level of special relativity first in the context of Abelian theories and later in non-Abelian theories. In the Abelian case the solitons are stabilized by the conservation of physical charges and in the non-Abelian theories by topological constraints.

At the general relativistic level less is known. In particular the influence of the gravitational field on the known special relativistic structures has not been extensively studied. There have been, however, many studies of boson stars, which present a similar formal problem, but concern stellar structure rather than elementary particles.

At the level of particle physics, one has an eigenvalue problem that attempts to model the elementary particles similar to the way that the Schrödinger equation models atoms and nuclei. In the $1 / N$ limit of standard theory Witten and collaborators have described a solitonic model of baryons. ${ }^{1}$

In examining string and supergravity theories from this viewpoint one finds two kinds of solitons. In the first class are the black-hole solitons which concentrate mass within a lump but are not true solitons since they carry central singularities and horizons. Typical of the second class are the topological solitons discovered by Strominger: these are five-branes immersed in a tendimensional space. Since they are singularity free they resemble solitons but are more precisely described as instantons, since their metric is Euclidean. Although nontopological solitons have not been found in these theories it is natural to ask if they exist in these or in other string or supergravity theories or in other completions of Einstein theory.

The present paper, a continuation of earlier exploratory studies along these lines, ${ }^{2}$ promotes the nonlinear spinor soliton to the general relativistic level. The original nonlinear spinor field, ${ }^{3}$

${ }^{\text {a)} E l e c t r o n i c ~ m a i l: ~ a c a d a v i d @ g a l i l e o . c s u n . e d u ~}$ 
characterized by a free spinor Lagrangian plus a quartic spinor interaction, was motivated by the then recently discovered universal Fermi interaction, and the conjecture that solitonic solutions of this equation might model the elementary particles. A current scenario might describe quarks and leptons while the quartic interaction would have to be mediated by heavy bosons. In the earlier model it turned out that this equation admitted only a small number of solitonic solutions in a way depending on the value of the "Fermi constant," which could be tuned to allow, for example, only three generations. Here we take the step of adding gravitational couplings to the original picture; and although the coupled spinor-gravitational field does not fall out of any of the currently favored fundamental theories, the solitons so defined should be of interest because of the fundamental character of both the spinor and gravitational fields.

The organization of the paper is as follows. In Sec. II the Lagrangian of the nonlinear spinor in a gravitational field is introduced. Sections III, IV, and V describe various basic aspects of the Kerr-Schild background including the effects of the Weyl rescaling of the metric with a dilation field. Sections VI-IX, introduce the spin connection, present the spinor field field equations, and display the contribution of the spinor to the energy momentum tensor. Section X discusses the full set of Einstein equations for a spinor in a Weyl rescaled Kerr-Schild background. Before attempting the solution of the full set of equations, we consider the special relativistic limit in Secs. XI and XII. Finally in Sec. XIII we present the results of the numerical treatment of the full set of equations. We end with a discussion of the results.

\section{FORMULATION}

We shall investigate solitons formed by the interaction of a nonlinear spinor field with the Einstein gravitational field. These two fields are to be codetermined by the following action:

$$
S=\int d^{4} x \sqrt{-g}(R-K L),
$$

where

$$
L=g^{\mu \lambda}: \bar{\psi} \gamma_{\mu} \nabla_{\lambda} \psi:-V(I)
$$

Here

$$
\nabla_{\lambda}=\partial_{\lambda}+\Gamma_{\lambda}
$$

where $\Gamma_{\lambda}$ is the spin connection satisfying

$$
\partial_{\lambda} \gamma_{\mu}-\Gamma_{\lambda \mu}^{\sigma} \gamma_{\sigma}+\left(\gamma_{\mu}, \Gamma_{\lambda}\right)=0
$$

in terms of the Christoffel connection $\Gamma_{\lambda \mu}^{\sigma}$. The notation: requires that the enclosed expression be symmetrized and Hermitized. The interaction $V(I)$, which may be rather general, may be chosen to generalize the universal Fermi interaction. For simplicity, however, we choose $I$ to be the scalar $\bar{\psi} \psi$.

Then the energy momentum tensor is

$$
\theta_{\mu \lambda}=\frac{\partial L}{\partial g^{\mu \lambda}}-\frac{1}{2} L g_{\mu \lambda}
$$

and the gravitational equations of motion are

$$
R_{\mu \lambda}=K \Theta_{\mu \lambda}, \quad K=-8 \pi \frac{k}{c^{2}},
$$

where $K$ is Newton's constant and 


$$
\Theta_{\mu \lambda}=\theta_{\mu \lambda}-\frac{1}{2} \theta g_{\mu \lambda} .
$$

By (2.2) one finds

$$
\Theta_{\mu \lambda}:=\bar{\psi} \gamma_{\mu} \nabla_{\lambda} \psi:-\frac{1}{2} V(I) g_{\mu \lambda} .
$$

In addition to the gravitational field equation (2.6) one has the following spinor field equation:

$$
g^{\mu \lambda} \gamma_{\mu} \nabla_{\lambda} \psi-\frac{\partial V}{\partial \bar{\psi}}=0
$$

The coupled equations (2.6) and (2.9) determine the structure of the soliton.

\section{KERR-SCHILD METRIC}

Solutions of the Dirac equation in the presence of an assigned time independent gravitational field have been discussed. These include solutions in a gravitational background produced by a rotating source such as a rotating star. ${ }^{4-6}$ For a rotating source the full Kerr-Schild background metric is required.

Here the role of the rotating source is played by the spinor field itself, since it necessarily carries angular momentum. The present problem is more difficult, however, than the problem of a rotating star since the spinor and gravitational fields are here codetermined while in the example of the star the gravitational field is a given background. On the other hand we know from the special relativistic problem ${ }^{3}$ that there are solitonic eigenfunctions of a spinor field in the background described by a spherical potential.

We shall therefore assume a spherically symmetric degenerate form of the Kerr-Schild gravitational field with the understanding that it may be necessary to add more structure in order to obtain a more refined solution.

\section{THE STATIC KERR-SCHILD METRIC}

Let us describe a gravitational field defined by the following Kerr-Schild metric:

$$
g_{\alpha \beta}=\eta_{\alpha \beta}-2 m \ell_{\alpha} \ell_{\beta} .
$$

Here $\eta_{\alpha \beta}$ is Minkowskian with the signature $(1,-1,-1,-1)$ and $\ell_{\alpha}$ is a null static field:

$$
\begin{aligned}
& \ell^{\alpha} \ell_{\alpha}=0, \\
& \frac{\partial \ell_{\alpha}}{\partial t}=0 .
\end{aligned}
$$

For this metric

$$
\operatorname{det} g=\operatorname{det} \eta=-1 \text {. }
$$

Set

$$
\ell^{\alpha}=\ell^{0}(1, \vec{\lambda}) .
$$

Then

$$
\lambda_{k} \lambda_{k}=1 \text {. }
$$

In the general case 


$$
\partial_{i} \lambda_{j}=\alpha P_{i j}+\beta \Lambda_{j i}
$$

where

$$
\begin{gathered}
P_{i j}=\delta_{i j}-\lambda_{i} \lambda_{j}, \\
\Lambda_{i j}=\epsilon_{i j k} \lambda_{k} .
\end{gathered}
$$

Both $\alpha$ and $\beta$ are harmonic functions. The function $\alpha$ may be regarded as a Newtonian potential in the presence of rotation, while $\beta$ is the specific angular momentum of the source. In both the rotating and nonrotating cases the $\lambda_{i}$ describe the paths followed by infalling particles. The divergence and curl of $\vec{\lambda}$ are determined by $\alpha$ and $\beta$, respectively.

At this point we leave the general formulation and focus in the spherically symmetric case. In this context $\lambda_{i}$ is given by

$$
\lambda_{i}=\frac{x_{i}}{r}, \quad r^{2}=x_{i} x_{i}
$$

Then

$$
\partial_{i} \lambda_{j}=\frac{1}{r}\left(\delta_{i j}-\lambda_{i} \lambda_{j}\right)
$$

and $\alpha$ is exactly the Newtonian potential while $\beta$ vanishes. Then also

$$
\begin{gathered}
\partial_{i} \lambda_{i}=\frac{2}{r}, \\
\partial_{i} \lambda_{j}-\partial_{j} \lambda_{i}=0, \\
\lambda^{i} \partial_{i} \lambda_{j}=\lambda^{i} \partial_{j} \lambda_{i}=0 .
\end{gathered}
$$

In the Newtonian case $\alpha$ is also $\ell_{0}^{2}$. It is useful to introduce two scalar fields, $C$ and $D$, by

$$
C \ell_{\alpha}=-\ell^{\mu} \partial_{\mu} \ell_{\alpha}
$$

and

$$
D=\partial_{\mu} \ell^{\mu} .
$$

Then

$$
\begin{gathered}
C=\lambda_{k} \partial_{k} \ell_{0}=\frac{d \ell_{0}}{d r}=\ell_{0}^{\prime} \\
D=\ell_{0}^{\prime}+\frac{2}{r} \ell_{0} .
\end{gathered}
$$

The Christoffel connection is a quadratic form in $m$ :

$$
\Gamma_{\alpha \beta}^{\mu}=\stackrel{1}{\Gamma} \Gamma_{\alpha \beta}^{\mu}+\stackrel{2}{\Gamma} \mu
$$

where 


$$
\stackrel{1}{\Gamma}_{\alpha \beta}^{\mu}=\frac{1}{2} \eta^{\mu \tau}\left(\partial_{\alpha} h_{\beta \tau}+\partial_{\beta} h_{\alpha \tau}-\partial_{\tau} h_{\alpha \beta}\right)
$$

and

$$
\stackrel{2}{\Gamma}_{\alpha \beta}^{\mu}=-4 m^{2} C \ell^{\mu} \ell_{\alpha} \ell_{\beta} .
$$

Here

$$
h_{\alpha \beta}=g_{\alpha \beta}-\eta_{\alpha \beta}=-2 m \ell_{\alpha} \ell_{\beta} .
$$

Also

$$
\begin{gathered}
\Gamma_{\mu \alpha}^{\mu}=0, \\
\ell_{\mu} \Gamma_{\alpha \beta}^{\mu}=-2 C \ell_{\alpha} \ell_{\beta}, \\
\ell^{\alpha} \Gamma_{\alpha \beta}^{\mu}=2 C \ell^{\mu} \ell_{\beta} .
\end{gathered}
$$

The Ricci tensor is

$$
\begin{gathered}
R_{00}=\left(2 m^{2} \varphi-m\right) \nabla^{2} \varphi, \\
R_{0 k}=2 m^{2}\left(\varphi \nabla^{2} \varphi\right) \lambda_{k}, \\
R_{j k}=\delta_{j k} \frac{2 m}{r}\left(\varphi^{\prime}+\frac{2 \varphi}{r}\right)+\lambda_{j} \lambda_{k}\left[m\left(\varphi^{\prime \prime}-\frac{4 \varphi}{r^{2}}\right)+2 m^{2} \varphi \nabla^{2} \varphi\right] .
\end{gathered}
$$

Here $\varphi=\ell_{0}^{2}$. The gravitational field described by the special assumptions that we have made is now defined completely by the function $\varphi(r)$ which is exactly the Newtonian potential if there is no rotation.

\section{THE RESCALED KERR-SCHILD METRIC}

Because of the back action of the spinor field acting through its energy momentum tensor it is necessary to introduce a second gravitational potential. To do this we rescale the Kerr-Schild metric by introducing the dilaton field, $\sigma(r)$, as follows:

$$
\bar{g}_{\alpha \beta}=e^{2 \sigma(r)} g_{\alpha \beta} .
$$

The rescaled connection is now

$$
\Gamma_{\alpha \beta}^{\mu}=\Gamma_{\alpha}^{\mu}+\delta_{\alpha}^{\mu} \partial_{\beta} \sigma+\delta_{\beta}^{\mu} \partial_{\alpha} \sigma-g_{\alpha \beta} g^{\mu \tau} \partial_{\tau} \sigma
$$

and the Ricci tensor then becomes

$$
\begin{gathered}
R_{\alpha \beta}=R_{\alpha \beta}-2 \sigma_{\alpha \beta}-\left[\Delta_{2} \sigma+2 \Delta_{1} \sigma\right] g_{\alpha \beta}, \\
\Delta_{1} \sigma=g^{\mu \lambda} \partial_{\mu} \sigma \partial_{\lambda} \sigma \\
\Delta_{2} \sigma=g^{\mu \lambda} \sigma_{, \mu \lambda}, \\
\sigma_{\mu \lambda}=\sigma_{, \mu \lambda}-\sigma_{, \mu} \sigma_{, \lambda}
\end{gathered}
$$


or

$$
R_{\mu \lambda}=R_{\mu \lambda}-\Delta_{\mu \lambda}
$$

where

$$
\Delta_{\mu \lambda}=2 \sigma_{\mu \lambda}+g_{\mu \lambda} \Delta_{c}
$$

and

$$
R_{\mu \lambda}=R_{\mu \lambda} \quad(\sigma=0) .
$$

One finds

$$
\begin{gathered}
\Delta_{00}=2 m(1-2 m \varphi) \varphi^{\prime} \sigma^{\prime}+(1-2 m \varphi) \Delta_{c}, \\
\Delta_{0 k}=\left\{-4 m^{2} \varphi \varphi^{\prime} \sigma^{\prime}-2 m \varphi \Delta_{c}\right\} \lambda_{k}, \\
\Delta_{j k}=\left[2(1-2 m \varphi) \frac{\varphi^{\prime}}{r}-\Delta_{c}\right] \delta_{j k} \\
+2\left[\sigma^{\prime \prime}-\frac{\sigma^{\prime}}{r}-\left(\sigma^{\prime}\right)^{2}-m\left(\varphi^{\prime}-\frac{2}{r} \varphi\right) \sigma^{\prime}-2 m^{2} \varphi \sigma^{\prime} \varphi^{\prime}-2 m \varphi \Delta_{c}\right] \lambda_{j} \lambda_{k},
\end{gathered}
$$

and

$$
\begin{gathered}
\Delta_{c}=\Delta_{2} \sigma+2 \Delta_{1} \sigma \\
\Delta_{2} \sigma=-\nabla^{2} \sigma+2 m \varphi \sigma^{\prime \prime}+2 m\left(\varphi^{\prime}+\frac{2}{r} \varphi\right) \sigma^{\prime}, \\
\Delta_{1} \sigma=(2 m \varphi-1)\left(\sigma^{\prime}\right)^{2} .
\end{gathered}
$$

The gravitational field is now defined by the two scalar functions $\varphi(r)$ and $\sigma(r)$. The Ricci tensor becomes

$$
\begin{gathered}
R_{00}=(\hat{\varphi}-1)\left[\frac{1}{2} \nabla^{2} \hat{\varphi}+\hat{\varphi}^{\prime} \sigma^{\prime}+\Delta_{c}\right], \\
R_{0 k}=\hat{\varphi}\left[\frac{1}{2} \nabla^{2} \hat{\varphi}+\hat{\varphi}^{\prime} \sigma^{\prime}+\Delta_{c}\right] \lambda_{k}, \\
R_{j k}=R^{a} \eta_{j k}+R^{b} \lambda_{j} \lambda_{k},
\end{gathered}
$$

where

$$
\begin{gathered}
R^{a}=-\frac{1}{r}\left(\hat{\varphi}^{\prime}+\frac{2}{r} \hat{\varphi}\right)-2(1-\hat{\varphi}) \frac{\sigma^{\prime}}{r}-\Delta_{c}, \\
R^{b}=\frac{1}{2} \hat{\varphi}^{\prime \prime}-\frac{1}{r^{2}} \hat{\varphi}+\frac{1}{2} \hat{\varphi} \nabla_{\hat{\varphi}}^{2}-2\left[\sigma^{\prime \prime}-\frac{\sigma^{\prime}}{r}-\left(\sigma^{\prime}\right)^{2}-\left(\frac{\hat{\varphi}^{\prime}}{2}-\frac{1}{r} \hat{\varphi}\right) \sigma^{\prime}-\frac{1}{2} \hat{\varphi} \hat{\varphi}^{\prime} \sigma^{\prime}-\hat{\varphi} \Delta_{c}\right], \\
\hat{\varphi}=2 m \varphi
\end{gathered}
$$




$$
\Delta_{c}=-\nabla^{2} \sigma+\hat{\varphi} \sigma^{\prime \prime}+\left(\hat{\varphi}^{\prime}+\frac{2}{r} \hat{\varphi}\right) \sigma^{\prime}+2(\hat{\varphi}-1)\left(\sigma^{\prime}\right)^{2}
$$

\section{THE SPIN CONNECTION}

The Dirac matrices corresponding to the Kerr-Schild metric (before rescaling) may be chosen as follows: ${ }^{5}$

$$
\gamma_{\alpha}=\eta_{\alpha}-\sqrt{2 m} \ell{ }_{\alpha} \eta_{5}
$$

where

$$
\begin{gathered}
\left(\eta_{\alpha}, \eta_{\beta}\right)_{+}=2 \eta_{\alpha \beta}, \\
\eta_{5}=\eta_{0} \eta_{1} \eta_{2} \eta_{3} .
\end{gathered}
$$

A choice more useful for computing the spin connection is ${ }^{7}$

$$
\gamma_{\alpha}=\eta_{\alpha}-m \ell_{\alpha} \hat{\tau}
$$

where

$$
\hat{\tau}=\ell^{\mu} \eta_{\mu}
$$

or

$$
\gamma_{\alpha}=e_{\alpha}^{\mu} \eta_{\mu}
$$

with

$$
e_{\alpha}^{\mu}=\delta_{\alpha}^{\mu}-m \ell_{\alpha} \ell^{\mu} .
$$

If $g_{\mu \lambda}$ is rescaled, then $\gamma_{\mu}$ must also be rescaled and

$$
\bar{\gamma}_{\mu}=\bar{e}_{\mu}^{-\lambda} \eta_{\lambda}
$$

where

$$
\bar{e}_{\mu}^{\lambda}=e^{\sigma(r)} e_{\mu}^{\lambda}
$$

while the new spin connection $\bar{\Gamma}_{\lambda}$ must satisfy

$$
\partial_{\lambda} \bar{\gamma}_{\mu}-\bar{\Gamma}_{\mu \lambda}^{\alpha} \bar{\gamma}_{\alpha}+\left(\bar{\gamma}_{\mu}, \bar{\Gamma}_{\lambda}\right)=0 .
$$

Here the Cristoffel connection $\bar{\Gamma}_{\mu \lambda}^{\alpha}$ is given by (5.2).

Let us set

$$
\begin{aligned}
& \Gamma_{\lambda}=g_{\lambda \alpha \beta}\left(\eta^{\alpha}, \eta^{\beta}\right)=2 g_{\lambda[\alpha \beta]} \eta^{\alpha} \eta^{\beta}, \\
& \bar{\Gamma}_{\lambda}=\bar{g}_{\lambda \alpha \beta}\left(\bar{\eta}^{\alpha}, \bar{\eta}^{\beta}\right)=2 \bar{g}_{\lambda[\alpha \beta]} \bar{\eta}^{\alpha} \bar{\eta}^{\beta} .
\end{aligned}
$$

Then (6.10) leads to the following relations:

$$
-8 \bar{g}_{\lambda[\beta \rho]}=\left(e^{-1}\right)_{\beta}^{\mu} \bar{\Gamma}_{\mu \lambda}^{\alpha} e_{\alpha \rho}+\left(e^{-1}\right)_{\beta}^{\mu} \partial_{\lambda} e_{\mu \rho}+\eta_{\rho \beta} \partial_{\lambda} \sigma .
$$


Since the second and third terms are symmetric in $\beta$ and $\rho$, they will cancel when multiplied by the commutator in (6.12) and therefore may be dropped. Then

$$
-8 \bar{g}_{\lambda[\beta \rho]}=\left(e^{-1}\right)_{\beta}^{\mu} \bar{\Gamma}_{\mu \lambda}^{\alpha} e_{\alpha \rho}
$$

Set

$$
\begin{gathered}
\bar{\Gamma}_{\alpha \lambda}^{\mu}=\Gamma_{\alpha \lambda}^{\mu}+\Delta_{\alpha \lambda}^{\mu}, \\
\bar{g}_{\lambda[\beta \rho]}=g_{\lambda[\beta \rho]}+E_{\lambda[\beta \rho]} .
\end{gathered}
$$

Then

$$
-4 E_{\lambda \beta \rho}=\left(e^{-1}\right)_{\beta}^{\mu} \Delta_{\mu \lambda}^{\alpha} e_{\alpha \rho}+\eta_{\beta \rho} \partial_{\lambda} \sigma
$$

where

$$
\Delta_{\mu \lambda}^{0}=\delta_{\mu}^{\alpha} \partial_{\lambda} \sigma+\delta_{\lambda}^{\alpha} \partial_{\mu} \sigma-g_{\mu \lambda} g^{\alpha \tau} \partial_{\tau} \sigma
$$

Since $E_{\lambda \beta \rho}$ is antisymmetric in $\beta$ and $\rho$, we may drop the second term in (6.17). Reduction of (6.14) and (6.17) gives

$$
\begin{gathered}
g_{\mu}^{\alpha \beta}=-\frac{m}{4} \partial^{\alpha}\left(\ell_{\mu} \ell^{\beta}\right), \\
E_{\mu}^{\alpha \beta}=\frac{1}{4}\left[e_{\mu}^{\alpha} \partial^{\beta} \sigma-\left(\ell^{\mu} \partial_{\mu} \sigma\right) \ell^{\alpha} \delta_{\mu}^{\beta}\right], \\
\bar{\Gamma}_{\mu}=\frac{1}{4}\left[-m \partial^{\alpha}\left(\ell_{\mu} \ell^{\beta}\right)+e_{\mu}^{\alpha} \partial^{\beta} \sigma-m \ell^{\alpha} \delta_{\mu}^{\beta} \nabla \sigma\right]\left(\gamma_{\alpha}, \gamma_{\beta}\right),
\end{gathered}
$$

where

$$
\nabla \sigma=\ell^{\alpha} \partial_{\alpha} \sigma
$$

\section{THE SPINOR FIELD EQUATIONS}

In the rescaled Kerr-Schild metric Eq. (2.12) becomes

$$
\bar{\gamma}^{\mu}\left(\partial_{\mu}+\bar{\Gamma}_{\mu}\right) \psi-\frac{\partial V}{\partial \bar{\psi}}=0
$$

where $\bar{\Gamma}_{\mu}$ is the spin connection and $\partial V / \partial \psi$ represents all the nonlinear interactions. We shall assume a four-component spinor and adopt the following ansatz:

$$
\psi=Z \Omega+W \beta \Omega
$$

with

$$
\Omega=\frac{1}{\sqrt{2}}\left(\begin{array}{c}
1 \\
0 \\
\lambda_{3} \\
\lambda_{+}
\end{array}\right), \quad \beta=\left(\begin{array}{cc}
I & 0 \\
0 & -I
\end{array}\right)
$$

when $\vec{\lambda}$ is the three-vector defined by (4.5) and $\lambda_{+}=\lambda_{1}+i \lambda_{2}$. If $Z$ and $W$ are complex conjugates so that 


$$
\begin{aligned}
& Z-\frac{1}{2}(F+i G), \\
& W=\frac{1}{2}(F-i G),
\end{aligned}
$$

then

$$
\psi=\frac{1}{\sqrt{2}} e^{i \omega t}\left(\begin{array}{c}
F \\
0 \\
i G \lambda_{3} \\
i G \lambda_{+}
\end{array}\right)=\frac{1}{\sqrt{2}} e^{i \omega t}\left(\begin{array}{c}
F \\
0 \\
i G \cos \theta \\
i G \sin \theta e^{i \phi}
\end{array}\right)
$$

If the ambient space is flat and $\psi$ is described by (7.5) then $\psi$ is also an eigenfunction of the Dirac angular momentum with spin $1 / 2$. Therefore in the limit of weak gravitational field we may require that $\psi$ approach $(7.5) .^{3}$

By $(6.5)$

$$
\begin{gathered}
\hat{\tau} \Omega=0, \\
\hat{\tau} \beta \Omega=2 \ell_{0} \Omega .
\end{gathered}
$$

Define

$$
\tau=\eta_{k} \lambda_{k}=-\eta^{k} \lambda_{k}
$$

then

$$
\begin{gathered}
\tau \Omega=\beta \Omega, \\
\tau \beta \Omega=-\Omega
\end{gathered}
$$

also

$$
\begin{gathered}
\hat{\tau}^{2}=0, \quad \tau^{2}=-1, \\
\frac{d \tau}{d r}=0 .
\end{gathered}
$$

The following identity is also useful:

$$
\eta_{s} \partial_{s}=\tau\left(\frac{d}{d r}+\frac{1}{r}\right)-\frac{1}{r} \tau \beta k,
$$

where

$$
k=\beta(\vec{\Sigma} \vec{L}+1), \quad \vec{L}=\vec{r} \times \vec{p} .
$$

Here

$$
\vec{\Sigma}=\left(\begin{array}{cc}
\vec{\sigma} & 0 \\
0 & \vec{\sigma}
\end{array}\right)
$$

Note also

$$
\begin{aligned}
& k \beta=\beta k, \\
& k \Omega=\Omega .
\end{aligned}
$$


To reduce (7.1) let us next compute

$$
\gamma^{\mu} \partial_{\mu} \psi=\eta^{\mu} \partial_{\mu} \psi+m \hat{\tau} \ell^{\mu} \partial_{\mu} \psi
$$

By (7.2) and (7.11) we find

$$
\begin{aligned}
\bar{\gamma}^{\mu} \partial_{\mu} \psi= & e^{\sigma}\left[(1-2 m \varphi) W^{\prime}+i \omega(1+2 m \varphi) W+(W-Z) \frac{1}{r}\right] \Omega \\
& -e^{\sigma}\left[Z^{\prime}-i \omega Z+(Z-W)\left(\frac{1}{r}\right)\right] \beta \Omega .
\end{aligned}
$$

By (6.8) and (6.21) the spin connection gives the contribution

$$
\bar{\gamma}^{\mu} \bar{\Gamma}_{\mu} \psi=\left(W f_{2} \Omega+Z f_{1} \beta \Omega\right) e^{\sigma},
$$

where

$$
\begin{gathered}
f_{1}=\frac{3}{2} \sigma^{\prime} \\
f_{2}=3 m \sigma^{\prime} \varphi-\frac{3}{2} \sigma^{\prime}-m\left(\varphi^{\prime}+\frac{2}{r} \varphi\right) .
\end{gathered}
$$

Let us choose the invariant nonlinear interaction to be a functional of

$$
\begin{aligned}
I & =\widetilde{\psi} \psi \\
& =\bar{S} W+Z \bar{W} .
\end{aligned}
$$

Then the nonlinear term in the equation of motion is

$$
\begin{aligned}
\frac{\partial V}{\partial \bar{\psi}} & =\frac{d V}{d I} \frac{\partial I}{\partial \bar{\psi}} \\
& =V^{\prime} \psi .
\end{aligned}
$$

For definiteness we take

$$
V(I)=m_{0} I+\frac{g}{2} I^{2}, \quad m_{0}>0, g<0 .
$$

Then

$$
\frac{\partial V}{\partial \bar{\psi}}=\left(m_{0}+g I\right) \psi=\left[m_{0} Z+g(\bar{Z} W+\bar{W} Z) Z\right] \Omega+\left[m_{0} W+g(\bar{Z} W+\bar{W} Z) W\right] \beta \Omega
$$

and the differential equation (7.1) becomes by (7.17) and (7.18)

$$
\mathcal{Z} \Omega+\mathcal{W}(\beta \Omega)=0
$$

where 


$$
\mathcal{Z}=(1-2 m \varphi) W^{\prime}+(W-Z) \frac{1}{r}+\left[i \omega(1+2 m \varphi)+f_{2}\right] W-e^{-\sigma}\left[m_{0}+g(\bar{Z} W+\bar{W} Z)\right] Z
$$

and

$$
\mathcal{W}=-Z^{\prime}+\left(i \omega+f_{1}\right) Z-(Z-W) \frac{1}{r}-e^{-\sigma}\left[m_{0}+g(\bar{Z} W+\bar{W} Z)\right] W
$$

The spinors $\Omega$ and $\beta \Omega$ are orthogonal:

$$
\Omega^{+}(\beta \eta)=0
$$

Then by (7.26)

$$
\mathcal{Z}=\mathcal{W}=0
$$

By (7.27) and (7.28) one sees that (7.30) implies two complex differential equations for $z$ and $w$ or four real equations for real components $\left(Z_{1}, Z_{2}, W_{1}, W_{2}\right)$.

\section{THE SOURCE OF THE GRAVITATIONAL FIELD}

The source of the gravitational field, the energy-momentum tensor of the spinor field, is described by (2.8).

Let us consider

$$
: \bar{\psi} \bar{\gamma}_{\alpha} \bar{\nabla}_{\beta} \psi:
$$

where $\bar{\psi}$ is the usual Dirac adjoint and where

$$
\nabla_{\beta}=\partial_{\beta}+\bar{\Gamma}_{\beta}
$$

Here $\bar{\Gamma}_{\beta}$ is the spin connection given by $(6.21)$.

Let us decompose the bilinear part of the energy-momentum tensor as follows:

$$
\begin{aligned}
& \stackrel{0}{\Theta \beta}_{\alpha \beta}=: \bar{\psi} \bar{\gamma}_{\alpha} \partial_{\beta} \psi:, \\
& \stackrel{1}{\Theta \beta}_{\alpha \beta}=: \bar{\psi} \bar{\gamma}_{\alpha} \bar{\Gamma}_{\beta} \psi:
\end{aligned}
$$

where $\psi$ is given by (7.2).

It turns out that all the matrix elements of (8.3) and (8.4) are expressible in terms of $\lambda_{k}$ and $\mu_{k}$ given by

$$
\lambda_{k}=\Omega^{\dagger} \eta_{k} \beta \Omega
$$

and

$$
\mu_{k}=\Omega^{\dagger} \eta_{k} \Omega
$$

Here $\lambda_{k}$ is the three-vector defined by (4.5) and alternatively expressed as

$$
\lambda_{k}=(\sin \theta \cos \phi, \sin \theta \sin \phi, \cos \theta)
$$

while $\vec{\mu}$ is an imaginary vector orthogonal to $\vec{\lambda}$ and given by 


$$
\mu_{k}=i \epsilon_{k s 3} \lambda_{s}
$$

One finds that the gravitational equations are of the form

$$
A \lambda_{k}+B \mu_{k}=0
$$

and since $\vec{\lambda}$ and $\vec{\mu}$ are orthogonal

$$
A=B=0 \text {. }
$$

The corresponding gravitational equations then reduce to

$$
B=\bar{W} Z-\bar{Z} W=0
$$

becomes a constraint on the components of the spinor $\psi$.

Equation (8.11) implies

$$
W=u Z
$$

where $u$ is a real function. One finds for the complete energy-momentum tensor:

$$
\begin{gathered}
\Theta_{\alpha \beta}=\Theta_{\alpha \beta}+\Theta_{\alpha \beta}-\frac{1}{2} V g_{\alpha \beta}, \\
\Theta_{00}=\frac{e^{\sigma}}{2}\left\{\left[\left(1-\frac{3 \hat{\varphi}}{2}+\varphi^{2}\right) \sigma^{\prime}+\frac{(\hat{\varphi}-1)}{2} \frac{\hat{\varphi}^{\prime}}{2}\right] \Delta+\left[\left(\frac{\hat{\varphi}}{2}-\frac{\hat{\sigma}^{2}}{2}\right) \sigma^{\prime}-\frac{\hat{\varphi} \hat{\varphi}^{\prime}}{4}\right] \Sigma\right\}-\frac{1}{2}(1-\hat{\varphi}) e^{2 \sigma} V, \\
\Theta_{0 j}=\left[\left\{\left[\frac{1}{2}\left(1-\frac{\hat{\varphi}}{2}\right) \Sigma^{\prime}+\frac{\hat{\varphi}}{2} \Delta^{\prime}\right]+\frac{\Delta}{4}\left[\frac{\hat{\varphi}^{\prime}}{2}(\hat{\varphi}-1)+\sigma^{\prime}\left(-3 \frac{\hat{\varphi}}{2}+\hat{\varphi}^{2}\right)\right]\right.\right. \\
\left.\left.+\frac{\Sigma}{4}\left[-\frac{\hat{\varphi}^{\prime}}{2}\left(\hat{\varphi}^{\prime}+1\right)+\sigma^{\prime}\left(1-\frac{\hat{\varphi}}{2}-\hat{\varphi}^{2}\right)\right]\right\} e^{\sigma}+\frac{1}{2} \hat{\varphi} V e^{2 \sigma}\right] \lambda_{j}
\end{gathered}
$$

after having dropped the $\mu_{j}$ term

$$
\begin{aligned}
\Theta_{j k}= & \left.\left\{\left[\left(\frac{\hat{\varphi}}{2}-1\right) \sigma^{\prime}+\frac{1}{r} \frac{\hat{\varphi}}{2}-\frac{2}{r}\right] \frac{\Delta}{2}+\left[\frac{\hat{\varphi}}{2} \sigma^{\prime}+\frac{1}{r} \frac{\hat{\varphi}}{2}\right] \frac{\Sigma}{2}\right\} e^{\sigma}-\frac{1}{2} V e^{2 \sigma}\right] \eta_{j k} \\
& +\left[\left\{\left[-\frac{\hat{\varphi}}{2} \Sigma^{\prime}+\left(1+\frac{\hat{\varphi}}{2}\right) \Delta^{\prime}\right]+\left[\frac{\hat{\varphi}}{2 r}-\frac{2}{r}+\frac{\hat{\varphi} \hat{\varphi}^{\prime}}{4}+\left(-1+\frac{\hat{\varphi}}{2}+\hat{\varphi}^{2}\right) \sigma^{\prime}\right] \frac{\Delta}{2}\right.\right. \\
& \left.\left.+\left[\frac{\hat{\varphi}}{2 r}-\left(1+\frac{\hat{\varphi}}{2}\right) \frac{\hat{\varphi}^{\prime}}{2}-\left(\frac{\hat{\varphi}}{2}+\hat{\varphi}^{2}\right) \sigma^{\prime}\right] \frac{\Sigma}{2}\right\} e^{\sigma}+\frac{1}{2} \hat{\varphi} V e^{2 \sigma}\right] \lambda_{j} \lambda_{k} .
\end{aligned}
$$

Here

$$
\begin{gathered}
\Sigma=\bar{\psi} \eta_{0} \psi=|Z|^{2}+|W|^{2}, \\
\Delta=|Z|^{2}-|W|^{2} .
\end{gathered}
$$

Let us also write

$$
\Theta_{j k}=\Theta^{a} \eta_{j k}+\Theta^{b} \lambda_{j} \lambda_{k}
$$




\section{REDUCTION OF THE SPINOR EQUATION}

Because of the constraint (8.12) $(W=u Z)$ it is now possible to show that one may reduce the equations (7.30) from a system of four real equations with four real functions to two real equations with two real functions.

Set

$$
\begin{aligned}
& \alpha=1-2 m \varphi, \\
& \beta=1+2 m \varphi .
\end{aligned}
$$

Then

$$
\begin{gathered}
\mathcal{Z}=\alpha W^{\prime}+\frac{1}{r}(W-Z)+\left[i \beta \omega+f_{2}\right] W-e^{-\sigma}\left[m_{0}+g(\bar{Z} W+\bar{W} Z)\right] Z=0, \\
\mathcal{W}=Z^{\prime}-\left(i \omega+f_{1}\right) Z-\frac{1}{r}(W-Z)+e^{-\sigma}\left[m_{0}+g(\bar{Z} W+\bar{W} Z)\right] W=0 .
\end{gathered}
$$

Equation (9.2) and (9.3) may be combined by eliminating $Z^{\prime} / Z$. Then

$$
\begin{aligned}
& \alpha u^{\prime}+\left[\alpha u^{2}+(1-\alpha) u-1\right] \frac{1}{r}+i \omega(\alpha+1) u+\left(f_{2}+\alpha f_{1}\right) u \\
& -e^{-\sigma}\left[m_{0}+g(\bar{Z} W+\bar{W} Z)\right]\left(1+\alpha u^{2}\right)=0 .
\end{aligned}
$$

Since all terms in this equation are real except $2 i \omega(\alpha+1) u$, it follows that $\omega=0$.

It then follows from (8.12) and (9.3) that $Z^{\prime} / Z$ is also real or that the argument of $Z$ is constant and may be equated to zero. Then $Z$ may be taken real, and we may therefore take both $W$ and $Z$ real in (9.2) and (9.3).

$$
\begin{gathered}
\alpha W^{\prime}+(W-Z) \frac{1}{r}+f_{2} W-e^{-\sigma}\left[m_{0}+2 g W Z\right] Z=0, \\
Z^{\prime}-f_{1} Z-(W-Z) \frac{1}{r}+e^{-\sigma}\left[m_{0}+2 g W Z\right] W=0 .
\end{gathered}
$$

\section{GRAVITATIONAL FIELD EQUATIONS}

The gravitational equations (2.6) may be reduced to the following four equations:

$$
\begin{gathered}
(1-\hat{\varphi})\left[-\frac{1}{2} \nabla^{2} \hat{\varphi}-\hat{\varphi}^{\prime} \sigma^{\prime}-\Delta_{c}\right]=K \Theta_{00}, \\
\hat{\varphi}\left[\frac{1}{2} \nabla^{2} \hat{\varphi}+\hat{\varphi}^{\prime} \sigma^{\prime}+\Delta_{c}\right] \lambda_{k}=K \Theta_{0 k}, \\
{\left[\frac{1}{r}\left(\hat{\varphi}^{\prime}+\frac{2 \hat{\varphi}}{r}\right)-2(1-\hat{\varphi}) \frac{\sigma^{\prime}}{r}+\Delta_{c}\right]=-K \Theta^{a},} \\
\frac{\hat{\varphi}^{\prime \prime}}{2}-\frac{2 \hat{\varphi}}{r^{2}}+\frac{1}{2} \hat{\varphi} \nabla^{2} \hat{\varphi}-2\left[\sigma^{\prime \prime}-\frac{\sigma^{\prime}}{r}-\left(\sigma^{\prime}\right)^{2}-\left(\frac{\hat{\varphi}^{\prime}}{2}-\frac{1}{r} \hat{\varphi}\right) \sigma^{\prime}-\frac{1}{2} \hat{\varphi} \hat{\varphi}^{\prime} \sigma^{\prime}-\hat{\varphi} \Delta_{c}\right]=K \Theta^{b},
\end{gathered}
$$


where $\hat{\varphi}=2 m \varphi$,

$$
\Theta_{j k}=\Theta^{a} \eta_{j k}+\Theta^{b} \lambda_{j} \lambda_{k} .
$$

$\Delta_{c}$ is given by (5.13), $\Theta$ by (8.14)-(8.16). By (10.1) and (10.2)

$$
\hat{\varphi} \Theta_{00}+\left(\Theta_{0 k} \lambda_{k}\right)(1-\hat{\varphi})=0 .
$$

To study these equations near the origin impose solitonic boundary conditions requiring that all fields be finite with flat tangents at the origin. Then

$$
\begin{gathered}
\hat{\varphi}^{\prime}(0)=\sigma^{\prime}(0)=Z^{\prime}(0)=W^{\prime}(0)=0, \\
\Delta_{c}(0)=\left[-\nabla^{2} \sigma+\hat{\varphi} \sigma^{\prime \prime}\right]_{r=0} .
\end{gathered}
$$

From (8.17)

$$
\Delta(0)=0, \quad \Sigma(0)=2 Z^{2} .
$$

By (8.14)-(8.17)

$$
\begin{gathered}
{\left[\Theta_{00}\right]_{r=0}=\frac{e^{2 \sigma}}{2}[\hat{\varphi}(0)-1] V[I(0)],} \\
{\left[\Theta_{0 k}\right]_{r=0}=\frac{e^{2 \sigma}}{2} V[I(0)] \hat{\varphi}(0) \lambda_{k},} \\
{\left[\Theta_{j k}^{a}\right]_{r=0}=\left[\frac{\Sigma}{4}\left(\frac{\hat{\varphi}}{r}\right) e^{\sigma}-\frac{e^{2 \sigma}}{2} V\right]_{r=0} \eta_{j k},} \\
{\left[\Theta_{j k}^{b}\right]_{r=0}=\left[\frac{\Sigma}{4}\left(\frac{\hat{\varphi}}{r}\right) e^{\sigma}+\hat{\varphi} e^{2 \sigma} \frac{V}{2}\right]_{r=0} \lambda_{j} \lambda_{k} .}
\end{gathered}
$$

By (10.3)

$$
\lim _{r \rightarrow 0}\left\{2 \hat{\varphi}(r)-2 r^{2}\left[(1-\hat{\varphi}(r)) \frac{\sigma^{\prime}(r)}{r}-\frac{\hat{\varphi}}{2} \sigma^{\prime \prime}+\frac{\nabla^{2} \sigma}{2}\right]\right\}=\lim _{r \rightarrow 0} \frac{K}{2} r^{2}\left(\hat{\varphi} \frac{\Sigma}{r}-e^{2 \sigma} V\right) .
$$

Therefore

$$
\hat{\varphi}(0)=0
$$

The vanishing of $\hat{\varphi}(r)$ at the origin now implies the additional reduction of (10.1)

$$
-\frac{1}{2} \hat{\varphi}^{\prime \prime}(0)+\sigma^{\prime \prime}(0)=-\frac{K}{2} e^{2 \sigma(0)} V[I(0)] .
$$

\section{THE SPECIAL RELATIVISTIC EIGENVALUE PROBLEM}

In a previous study of a nonlinear scalar field coupled to the gravitational field it was found that the eigensolutions of the complete set of differential equations is critically dependent on the behavior of the nonlinear component alone. By following the earlier argument we shall now find, however, that the pattern for the nonlinear spinor problem is quite different. 
As before, we begin by associating an approximately conserved function with the nonlinear equations after they have been decoupled from the gravitational field. In this special relativistic limit where $(m=0, \sigma=0)$ we have $f_{1}=f_{2}=0$. After deletion of the mass and nonlinear coupling the two equations (9.5) and (9.6) become

$$
\begin{aligned}
& W^{\prime}-\frac{d V}{d I} Z+\frac{W-Z}{r}=0, \\
& Z^{\prime}+\frac{d V}{d I} W-\frac{W-Z}{r}=0 .
\end{aligned}
$$

We consider the auxiliary equations obtained by deleting the explicit dependence on $r$,

$$
\begin{aligned}
& W^{\prime}-\mathcal{V} Z=0, \\
& Z^{\prime}+\mathcal{V} W=0,
\end{aligned}
$$

where

$$
\mathcal{V}=\frac{d V}{d I} .
$$

Any invariant function $(\mathcal{F})$ associated with these equations satisfies

$$
\mathcal{F}^{\prime}=\frac{\partial \mathcal{F}}{\partial W} W^{\prime}+\frac{\partial \mathcal{F}}{\partial Z} Z^{\prime}=0
$$

or

$$
\mathcal{F}^{\prime}=\mathcal{V}\left(W \frac{\partial}{\partial Z}-Z \frac{\partial}{\partial Z}\right) \mathcal{F}=0
$$

Hence if $F$ is a functional of $W^{2}+Z^{2}$, it will be conserved for any solution of the set (11.3), (11.4). For the complete equations (11.1) and (11.2) however, we have

$$
\begin{aligned}
\mathcal{F}^{\prime} & =\frac{\partial \mathcal{F}}{\partial W}\left(\mathcal{V} Z+\frac{Z-W}{r}\right)+\frac{\partial \mathcal{F}}{\partial Z}\left(-\mathcal{V} W-\frac{Z-W}{r}\right)=\left(\frac{\partial \mathcal{F}}{\partial W}-\frac{\partial \mathcal{F}}{\partial Z}\right) \frac{Z-W}{r} \\
& =2(W-Z) \frac{\partial \mathcal{F}}{\partial \Sigma}\left(\frac{Z-W}{r}\right) .
\end{aligned}
$$

Then

$$
\mathcal{F}^{\prime}=-2 \frac{\partial \mathcal{F}}{\partial \Sigma}(W-Z)^{2}
$$

In the simplest case

$$
\mathcal{F}(\Sigma)=\Sigma \text {. }
$$

Then

$$
\mathcal{F}^{\prime}=-\frac{2(W-Z)^{2}}{r} \leqslant 0 .
$$


According to (11.3) and (11.4) the representative point moves in the circle ( $\Sigma=$ const) in the $W-Z$ plane. According to (11.1) and (11.2) the motion of this point deviates from the circle to satisfy (11.12), which directs the motion always toward lower values of $F$, i.e., toward the origin. The representative point will therefore spiral into the origin.

If the corresponding curve in configuration space represents an eigensolution it must satisfy the following boundary conditions at the origin and at $\infty$ :

At $r=0$,

$$
W^{\prime}=Z^{\prime}=0
$$

and at $r=\infty$,

$$
W^{\prime}=Z^{\prime}=W=Z=0 \text {. }
$$

To satisfy these boundary conditions on Eqs. (11.1) and (11.2) the eigensolution must begin at a point on the line $W=Z$ and it must end at the origin.

For the particular choice of (2.9) we have

$$
I=2 Z W
$$

The $\mathcal{V}$ is constant on the hyperbolas

$$
W Z=\text { constant }
$$

and along the asymptote $\mathcal{V}$ is a function of $Z^{2}$.

In an earlier discussion of the special relativistic limit the Dirac spinor was taken to be (7.5). As long as there is no gravitational coupling there is no problem with this ansatz, and if one chooses $V$ to be given by (7.25) one finds according to Eqs. (16a), (16b) of Ref. 3

$$
\begin{gathered}
F^{\prime}+\mu G+2 \gamma\left(G^{2}-F^{2}\right) G=0, \\
G^{\prime}+\mu F+\frac{2}{r} G+2 \gamma\left(G^{2}-F^{2}\right) F=0,
\end{gathered}
$$

where we also set $\omega=0$ and $\mu$ and $\gamma$ are the. Here $F$ and $G$ are the large and small components of $\psi$ as given by (7.5). The dissipative function $\bar{H}$ associated with these equations is

$$
\bar{H}=\mu\left(G^{2}-F^{2}\right)+\gamma\left(G^{2}-F^{2}\right)^{2}
$$

leading to a phase portrait with two attractions.

To compare (11.18) and (11.19) with the present equations (11.1) and (11.2) set

$$
\begin{aligned}
& f=W+Z, \\
& g=W-Z .
\end{aligned}
$$

Then (11.1) and (11.2) become

$$
\begin{gathered}
f^{\prime}+\mathcal{V} g=0, \\
g^{\prime}-\mathcal{V} f+\frac{2 g}{r}=0,
\end{gathered}
$$

where

$$
\mathcal{V}=m_{0}+\gamma I
$$


and

$$
I=2 W Z+\frac{1}{2}\left(f^{2}-g^{2}\right)
$$

Then

$$
\begin{gathered}
f^{\prime}+m_{0} g+\frac{\gamma}{2}\left(f^{2}-g^{2}\right) g=0, \\
g^{\prime}-m_{0} f+\frac{2 g}{2}-\frac{\gamma}{2}\left(f^{2}-g^{2}\right) f=0 .
\end{gathered}
$$

Here $f$ and $g$ are large and small components in (11.26) and (11.27) just as $F$ and $G$ are large and small components in (11.17) and (11.18).

There is a term by term correspondence between the pair (11.26), (11.27) and the pair (11.17), (11.18) except for signs. The difference in signs, however, gives rise to a completely different phase portrait for the dissipative function in the two cases. In particular, there is only one attractor associated with (11.26) and (11.27) while there are two attractors in the other case.

The distinction between the two cases may be pushed a little further as follows.

The dissipative function for the $(f, g)$ pair may be chosen as a function of $\left(f^{2}+g^{2}\right)$ since

$$
\frac{d}{d r}\left(f^{2}+g^{2}\right)=-\frac{4 g^{2}}{r} \leqslant 0
$$

by (11.26) and (11.27). The corresponding argument for the $(F, G)$ pair yields

$$
\frac{d}{d r}\left(F^{2}-G^{2}\right)=-\frac{4 G^{2}}{r} \leqslant 0 .
$$

Therefore one may choose a function of $f^{2}+g^{2}$ in one case and of $F^{2}-G^{2}$ in the other to qualify as a dissipative function. In the $(F, G)$ case we chose the dissipative function to be the Hamiltonian of the associated mechanical problem (with the explicit $r$ term deleted), i.e., the Hamiltonian equations corresponding to the Hamiltonian (11.19) are the differential equations (11.17) and (11.18) where $G$ plays the role of the momentum. The corresponding step with the $(f, g)$ pair is not possible because (11.26) and (11.27) are not derivable from a Hamiltonian as one may see as follows.

If a Hamiltonian did exist for this set then we would have

$$
\begin{gathered}
\frac{\partial^{2} H}{\partial q \partial p}=\frac{\partial \dot{q}}{\partial q}(q, p), \\
\frac{\partial^{2} H}{\partial p \partial q}=-\frac{\partial \dot{p}}{\partial p}(q, p),
\end{gathered}
$$

or

$$
\frac{\partial \dot{q}(p, q)}{\partial q}=-\frac{\partial \dot{q}(q, p)}{\partial p}
$$

Since $f$ and $g$ play the roles of the generalized coordinate and momentum, respectively, and since $r$ plays the role of the time, the condition (11.31) reads for the pair $(f, g)$,

$$
\frac{\partial f^{\prime}(f, g)}{\partial f}=-\frac{\partial g^{\prime}(f, g)}{\partial g} .
$$


Since this relation is not satisfied for Eqs. (11.26) and (11.27), there is no corresponding Hamiltonian.

Additionally one cannot retain the $(F, G)$ description with (8.11) since the pair (8.11), (7.4), implies

$$
Z^{2}=W^{2}
$$

and therefore either $F=0$ or $G=0$.

\section{BEHAVIOR AT LARGE $r$}

At large $r$ we require that all functions and their first and second derivatives vanish. We also drop all terms in $\Theta_{\mu \nu}$ of order $1 / r$. Then by (10.1)-(10.4) in the limit

$$
\Theta_{\mu \nu}=0
$$

and by $(8.15)-(8.17)$

$$
V(I)=0 .
$$

By (7.25) and the preceding equation

$$
I=0, \frac{2 \mu}{\gamma} .
$$

Let us choose $I=0$. Then

$$
\lim _{r \rightarrow 0}\left(f^{2}-g^{2}\right)=0
$$

by (11.26).

In the limit (11.27) and (11.28) become

$$
\begin{gathered}
f^{\prime}+\mu g=0, \\
g^{\prime}-\mu f+\frac{2 g}{r}=0,
\end{gathered}
$$

or

$$
g^{\prime \prime}+\frac{2}{r} g^{\prime}+\mu^{2} g-\frac{2}{r^{2}} g=0 .
$$

$f$ and $g$ are of opposite parity and since $f(0) \neq 0$ we take $f$ even. Then to order $1 / r$,

$$
f=\frac{\sin \mu \nu}{r}
$$

and

$$
g=\frac{\cos \mu \nu}{r} .
$$

In contrast to this behavior the $(F, G)$ pair approach zero as $e^{\mu \nu} / r$. 


\section{SOLUTIONS OF THE GRAVITATIONAL FIELD EQUATIONS}

First we consider the solutions to the gravitational field equations $(10.1)-(10.5)$ when the matter fields are set to zero.

Equation (10.1) gives

$$
\hat{\varphi}^{\prime \prime}-(1-2 \hat{\varphi}) \sigma^{\prime \prime}=-\frac{2}{r} \hat{\varphi}^{\prime}-4 \sigma^{\prime} \hat{\varphi}^{\prime}+\frac{2}{r}(1-2 \hat{\varphi}) \sigma^{\prime}+2(1-2 \hat{\varphi})\left(\sigma^{\prime}\right)^{2} .
$$

Multiplying (10.5) by $\lambda^{j} \lambda^{k}$ and contracting we obtain

$$
(1+2 \hat{\varphi}) \hat{\varphi}^{\prime \prime}-\left(3-4 \hat{\varphi}^{2}\right) \sigma^{\prime \prime}=-\frac{2}{r}(1+2 \hat{\varphi}) \hat{\varphi}^{\prime}-(4+8 \hat{\varphi}) \sigma^{\prime} \hat{\varphi}^{\prime}+\frac{1}{r}\left(2-8 \hat{\varphi}^{2}\right) \sigma^{\prime}-8 \hat{\varphi}^{2}\left(\sigma^{\prime}\right)^{2} .
$$

The preceding two equations can be combined to solve for $\hat{\varphi}^{\prime \prime}$ and $\sigma^{\prime \prime}$ :

$$
\sigma^{\prime \prime}=\left(\sigma^{\prime}\right)^{2}
$$

and

$$
\hat{\varphi}^{\prime \prime}=-\frac{2}{r} \hat{\varphi}^{\prime}-4 \sigma^{\prime} \hat{\varphi}^{\prime}+\frac{2}{r}(1-2 \hat{\varphi}) \sigma^{\prime}+3(1-2 \hat{\varphi})\left(\sigma^{\prime}\right)^{2} .
$$

Equation (13.3) gives a solution of the form

$$
\sigma \sim-\log (r),
$$

which when substituted into (13.4) leads to

$$
\hat{\varphi} \sim \pm r^{2} .
$$

It is apparent that the coupling of the dilaton field with the Kerr-Schild scalar does not give a finite theory. We have proceeded to study the full set of equations (10.1)-(10.5), including the matter fields, in order to find out if these fields provide the necessary attractive properties to yield finite soliton type solutions.

Once again we selected Eq. (10.1) and the contraction of Eq. (10.5) with $\lambda^{j} \lambda^{k}$,

$$
\begin{aligned}
& \sigma^{\prime \prime}=\left(\sigma^{\prime}\right)^{2}-K \frac{e^{\sigma}}{4}\left[4 Z^{\prime} Z-4 W^{\prime} W(1+2 \hat{\varphi})+Z^{2}\left(\sigma^{\prime}-4 \frac{\hat{\varphi}^{\prime} \hat{\varphi}}{(1-2 \hat{\varphi})}\right)+W^{2} \sigma^{\prime}(1-2 \hat{\varphi})\right], \\
\hat{\varphi}^{\prime \prime}= & -\frac{2}{r} \hat{\varphi}^{\prime}-4 \sigma^{\prime} \hat{\varphi}^{\prime}+\frac{2}{r}(1-2 \hat{\varphi}) \sigma^{\prime}+3(1-2 \hat{\varphi})\left(\sigma^{\prime}\right)^{2}-K \frac{e^{\sigma}}{4}\left[Z^{2}\left[(3-2 \hat{\varphi}) \sigma^{\prime}-4 \frac{(1-\hat{\varphi})}{(1-2 \hat{\varphi})} \hat{\varphi}^{\prime}\right]\right. \\
& \left.+W^{2}\left[\left(4 \hat{\varphi}^{2}-1\right) \sigma^{\prime}+2 \hat{\varphi}^{\prime}\right]+2 Z^{\prime} Z(1-2 \hat{\varphi})+2 W^{\prime} W\left(4 \hat{\varphi}^{2}-1\right)-2 e^{\sigma} V\right] .
\end{aligned}
$$

To look for numerical solutions it is necessary to recast Eqs. (9.6), (9.7), (13.7), and (13.8) in dimensionless form. This was accomplished by introducing a fundamental length $r_{0}$ to rescale the variable $r$ and a fundamental field strength $\psi_{0}$ to rescale the fermionic fields. The equations of interest depend on three parameters: $\beta=m_{0} r_{0}^{2}, \gamma=g r_{0}^{2} \psi_{0}^{2} / 2$ and $\delta=K \psi_{0}^{2}$. To validate the numerical analysis we used two different methods: the MATLAB differential equation suite ${ }^{8}$ and the subroutine SDRIV2 from the SLATE package. ${ }^{9}$

We explored a large portion of the three-dimensional parameter space looking for finite solutions for the fields $\sigma, \hat{\varphi}, W$, and $Z$. By systematically exploring the three-dimensional parameter 

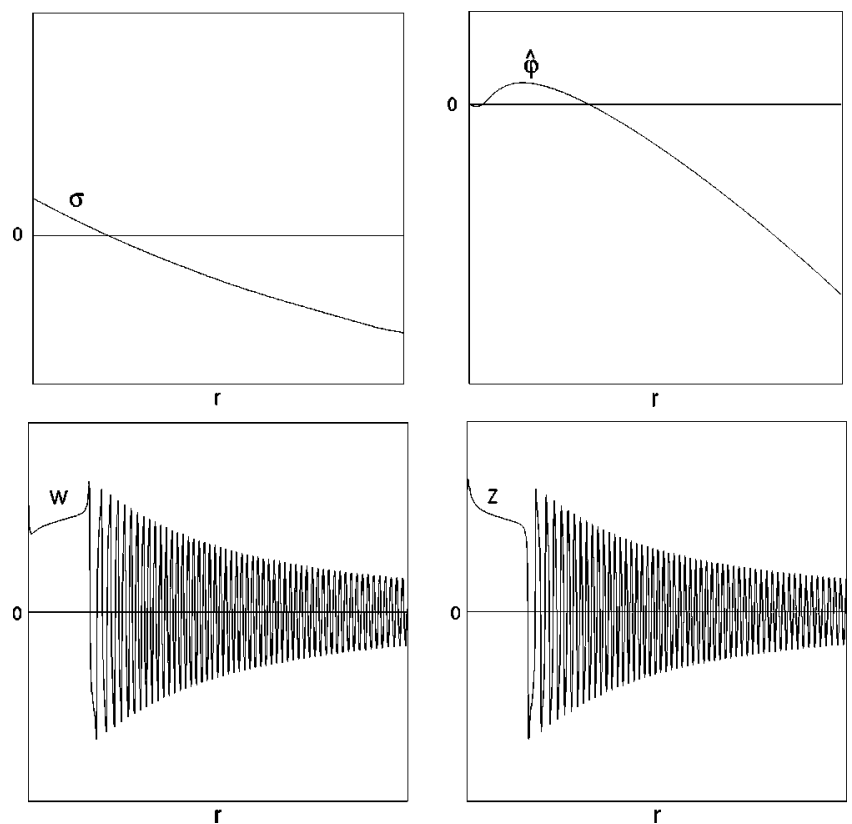

FIG. 1. Type A solution with nonsingular fields at finite $r$. The parameter values are: $\beta=0.044, \gamma=14.257$, and $\delta$ $=-0.001$ (since $K$ is negative).

space two types of solutions were encountered. The solutions of type A are shown in Fig. 1. While the fermion fields settle into the damped oscillations which characterize the special relativistic solutions, the fields $\sigma$ and $\hat{\varphi}$, while finite at small $r$, tend to $-\infty$ for large values of $r$. These solutions are characterized by a strong coupling for the four fermion interaction $(\gamma)$ in comparison to the mass $(\beta)$ and the fermion field strength $(\delta)$. The values of the parameters for the solutions displayed in the figure are: $\beta=0.044, \gamma=14.257$, and $\delta=-0.001$ (since $K$ is negative). We have not discovered a systematic relation between the values of the parameters that lead to a solution of type A. However the general pattern is $\delta \ll \beta \ll \gamma$. The other solution encountered, that we identify as type B, presents damped oscillations for the fermion fields while the $\sigma$ and $\hat{\varphi}$ fields are positive and monotonically increasing. This solution becomes singular at finite $r$ when the term $(2 \hat{\varphi}$ -1 ), in Eqs. (9.5), (13.7) and (13.8), vanishes. We did not find any systematic relation between the relative values of the parameters that lead to the solution of type B. Since the equations are nonlinear, the solutions are very sensitive to the parameter values. A change in the fourth significant figure in one of the parameters can lead to a change in the output from a type A to a type B solution.

From the numerical analysis it appears that the gravitational sector dominates and the fields $\sigma$ and $\hat{\varphi}$ follow the general trend of Eqs. (13.5) and (13.6) when there is no coupling to matter. While the numerical work performed does not constitute an exhaustive proof for the nonexistence of finite solutions as $r \rightarrow \infty$, it appears to be unlikely that there is a combination of parameters that would lead to such solutions.

\section{DISCUSSION}

It is known that at least two independent radial functions are needed for the solitonic solutions of the field equations that describe gravitational and scalar fields. ${ }^{7}$ There are therefore two independent radial functions in our ansatz for the present problem in which the gravitational field is coupled to a Dirac field. In our case, however, the second (Weyl) field would be described as a dilaton if it were localized.

In the original special relativistic problem it was found that there were no solitons for arbitrary values of the "Fermi coupling constant." In fact even when there were, there were only a few 
eigensolutions of the nonlinear equation. Initially encouraged by this result we set to look for numerical solutions of the equations for the gravitational and fermion fields. We explored systematically a large portion of the three-dimensional parameter space and only encountered the singular solutions of type A and B described in sec. XIII. While the solutions of type B are very common, those of type A occur for a particular ordering of the relative strengths of the parameters. We found that a variation in the fourth significant figure in any of the parameters could lead to a "jump" from a type A to a type B solution. While this does not constitute a definite proof of the nonexistence of finite solutions we think that it is unlikely that any combination of parameters may yield a result different from the singular solutions of type A and B.

In light of our failure to find a localized soliton in an earlier study, ${ }^{2}$ it may be further argued that the choice of a dilaton field for the second function makes a solution unlikely. In that work we proved that there are soliton solutions of the interacting gravitational, electromagnetic, scalar, and dilaton fields only if the dilaton field is excluded. Aside from the problem of the dilaton we have also shown (in Secs. XI and XII) that the non linear spinor field behaves quite differently in the special relativistic and general relativistic problems. For these reasons we now believe that the rescaled and spherically degenerate Kerr-Schild metric probably does not admit a spinor solution.

We have studied only the spherically problem. There is then the possibility that one would find a solitonic solution if the full angular dependence of the Kerr solution were restored. In fact the Kerr background was chosen in the first place to allow for the backaction on the gravitational field of the angular momentum carried by the spinor field.

Our original strategy was based on the expectation that there were solitons in the spherically symmetric background, as there are in the special relativistic problem, and that these solutions could then be improved by perturbatively taking into account the angular dependence of the Kerr geometry. In view of the present results this strategy no longer seems promising.

${ }^{1}$ G. S. Adkins, C. R. Nappi, and E. Witten, Nucl. Phys. B 228, 552 (1983).

${ }^{2}$ A. C. Cadavid and R. J. Finkelstein, Phys. Rev. D 57, 7318 (1998).

${ }^{3}$ R. Finkelstein, R. LeLevier, and M. Ruderman, Phys. Rev. 83, 326 (1951).

${ }^{4}$ S. A. Tevkolsky, Astrophys. J. 185, 635 (1973).

${ }^{5}$ S. Einstein and R. Finkelstein, J. Math. Phys. 18, 664 (1977).

${ }^{6}$ S. Chandrasekhar, Proc. R. Soc. London 349, 571 (1976).

${ }^{7}$ H. B. Joutei and A. Chakrabarti, Phys. Rev. D 19, 457 (1979).

${ }^{8}$ MATLAB Version 5.2. The MathWorks Inc.

${ }^{9}$ B. L. Buzbee, Sources and Development of Mathematical Software, the SLATEC Common Math. Library, edited by W. Cowell (Prentice-Hall, Englewood Cliffs, NJ, 1984). 